\title{
1.5 Port Vector Spectrometer for Terahertz Time Domain Spectroscopy
}

\author{
Fahd R. Faridi, Uttam Nandi, and Sascha Preu \\ Technische Universität Darmstadt, Darmstadt, Germany
}

\begin{abstract}
We demonstrate a spectrometer capable of measuring terahertz time domain transmission and reflection spectroscopy simultaneously in a single setup. This facilitates highly accurate determination of optical material parameters and the geometric thickness by taking advantage of both geometries. The system is also suitable for parameter extraction for materials when data from transmission geometry cannot be extracted and for characterizing non-time-invariant devices such as optical isolators.
\end{abstract}

(C) 2018 IEEE. Published version at:

https://ieeexplore.ieee.org/abstract/document/8510415

\section{INTRODUCTION}

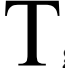
ERAHERTZ time domain spectroscopy (THz TDS) has gained popularity in the last few decades due to its ability for non-contact, non-invasive and non-destructive characterization of materials in a broad spectral range covering several THz. A single measurement in transmission THz TDS or reflection $\mathrm{THz}$ TDS contains relative phase information as well as transmittance or reflectance. Furthermore Fabry-Pérot (FP)-echo pulses that is the result of multiple reflections from the sample facets contain additional information on thickness and absorption. All these information can be used in data analysis to simultaneously determine the samples thickness and the dielectric optical parameters [1], [2].

Transmission THz TDS is typically used for characterization of non-opaque samples. For highly absorptive materials this technique is not suitable to extract all necessary information. Such materials can be characterized by reflection THz TDS [3]. However, this method features less precision. Firstly, the phase of the reflected signal, given by the Fresnel reflection coefficient, is much smaller compared to the one measured in transmission. As for the case of transmission the phase results from the propagation through the whole thickness of the sample [4]. Secondly, reflection THz TDS is very sensitive to the relative position of the sample compared to a reference mirror necessary to obtain a reference spectrum [5]. These limitations accumulate to a less precise characterization of materials for reflection $\mathrm{THz}$ TDS. The geometric thickness determined by only reflection measurements is afflicted by large errors.

The proposed spectrometer overcomes insufficiencies of individual transmission and reflection TDS measurements and provides more accurate results in versatile applications. In this paper we concentrate on one of the applications, namely, the determination of thickness and complex refractive index of highly absorbing materials. Data extracted from simultaneous transmission and reflection measurements give access to more information regarding the sample compared to individual ones. This, in turn, can be utilized for data analysis to obtain more accurate material parameters.

\section{METHODOLOGY}

In case of $\mathrm{THz}$ TDS, the sample is characterized by measuring the complex transmission or reflection coefficient or
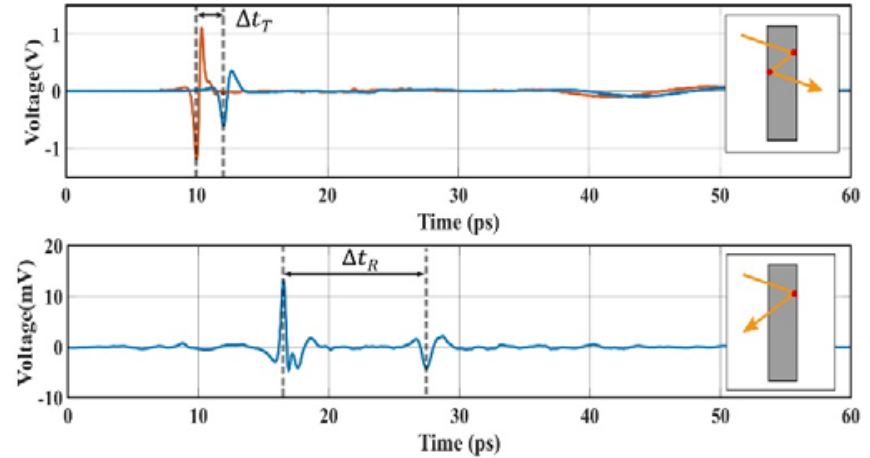

Fig. 1. Time Domain Terahertz Pulse of Reference (orange) and Polyvinyl Chloride (PVC) (blue) and THz path of the first echo through the sample (inset) for Transmission THz TDS (Top) and Reflection THz TDS (Bottom). The echo of the transmitted pulse is too weak for parameter extraction.

transfer function contains both amplitude and phase. For transmission TDS it is the ratio between the complex spectrum of the $\mathrm{THz}$ pulse propagating through the sample and reference $\mathrm{THz}$ spectra, where for reference the $\mathrm{THz}$ spectrum is recorded without any sample present. For reflection TDS it is the ratio of complex spectra reflecting from the sample and reference $\mathrm{THz}$ spectrum obtained by replacing the sample by a brass mirror. Analytically solving the equation of this transfer function in terms of amplitude and phase, gives the expressions for refractive index and absorption coefficient for both transmission TDS and reflection TDS [4]. However, the determination of these parameters, like in most of the spectroscopic methods, highly rely on the precise knowledge of the sample thickness. It is essential to determine the thickness along with the other parameters as suggested in the literature [1], [2]. For that, recording of FP echoes is required.

As far as high absorbing materials are concerned, such as thick samples of Polyvinyl chloride (PVC), the FP echoes are not or only hardly visible in transmission THz TDS, making it inadequate for thickness determination (Figure 1, top). Reflection TDS may still give access to the reflected pulse (Figure 1, bottom). As shown by the insets, the first echo in transmission geometry originates from a beam that goes through the absorptive material three times, and is reflected and transmitted twice. For the reflection geometry, the beam only travels twice through the material and is reflected only once and transmitted twice. One extra trip through a highly absorptive material along with one extra reflection in transmission TDS compared to reflection TDS reduces the signal amplitude severely, making it very hard to detect.

An algorithm is developed to simultaneously determine the thickness and optical parameters of a highly absorptive material using the data acquired from the 1.5 port vector spectrometer. As the first step, a preliminary thickness $(d)$ is estimated from the position of the peaks. From transmission TDS, the time delay between the reference pulse and the first transmitted pulse 
is given by

$$
\Delta t_{T}=(\bar{n}-1) d / c_{0}
$$

where, $\bar{n}$ is the average refractive index and $c_{0}$ is the speed of light in vacuum. Here we consider that the refractive index of air or dry nitrogen is 1 . From the reflection measurement, the time delay between first reflected pulse and first echo is

$$
\Delta t_{R}=2 \bar{n} d / c_{0}
$$

Solving Equation (1) and (2) we can estimate the value of $d$ and $\bar{n}$. Then the real and imaginary part of the refractive index, $n(\omega)$ and $\kappa(\omega)$, are estimated using the following equations

$$
\begin{aligned}
& n(\omega)=1-\frac{c_{0}}{\omega d} \angle T_{\text {exp }}(\omega) \\
& \kappa(\omega)=\frac{c_{0}}{\omega d}\left\{\ln \left[\frac{4 n(\omega)}{(1+n(\omega))^{2}}\right]-\ln \left|T_{\text {exp }}(\omega)\right|\right\}
\end{aligned}
$$

where, $\angle T_{\text {exp }}(\omega)$ and $\left|T_{\text {exp }}(\omega)\right|$ are the phase and amplitude of the experimentally obtained transfer function $T_{\exp }(\omega)$ [6].

After this initial evaluation of $d, n(\omega)$ and $\kappa(\omega)$, the transfer function, $T_{\text {calc }}(\omega)$, is calculated and compared to the experimental transfer function $T_{\exp }(\omega)$. In the next step, we minimize the function $\Delta T=\sum_{\omega}\left|T_{\text {calc }}(\omega)-T_{\exp }(\omega)\right|$ with numerical optimization of $n(\omega), \kappa(\omega)$ and $d$.

\section{EXPERIMENTAL VALIDATION}

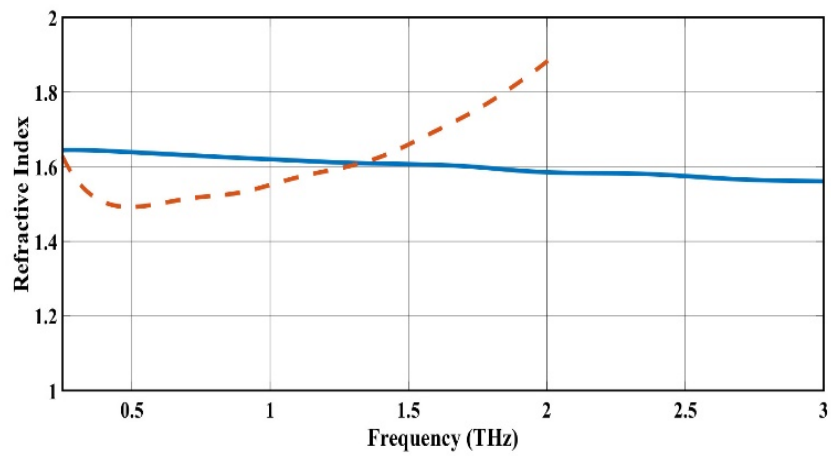

Fig. 2. Refractive Index of PVC calculated by using a 1.5 port vector spectrometer (blue) and only reflection THz TDS (orange dotted)

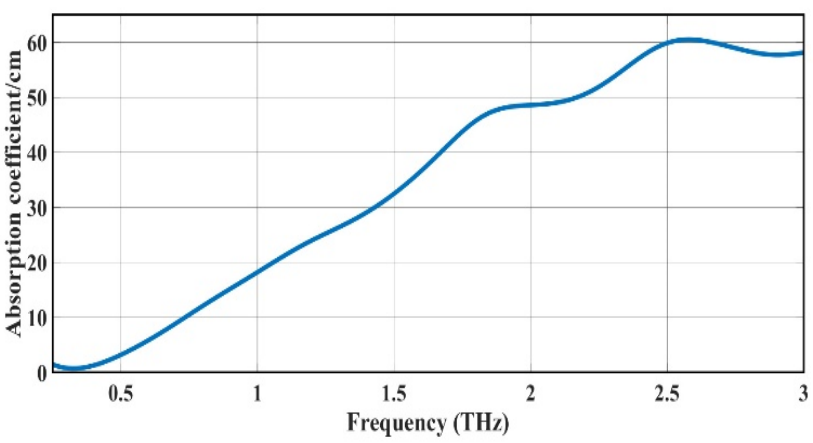

Fig. 3. Absorption Coefficient of PVC calculated by using 1.5 port vector spectrometer.

A mode-locked fiber laser from Menlo Systems is used to produce optical pulses with a pulse duration of $90 \mathrm{fs}$ and a repetition rate of $100 \mathrm{MHz}$. Laser pulses from the two fiber ports of the system drive transmitter and the receiver of the transmission TDS. A free space port, phase locked with the fiber ports, drives the reflection TDS receiver. An ErAs: $\operatorname{In}(\mathrm{Al}) \mathrm{GaAs}$ based photoconductive slotline antenna generates the THz pulses. For detection ErAs:InGaAs based photoconductive $\mathrm{H}$-dipole antennas are implemented similar to those in [7]. A delay stage is included in each receiver arm and wire grid polarizers split the reflected $\mathrm{THz}$ pulse coming from the sample towards the receiver of the reflection TDS.

The device under test for validating the material characterization aspect of 1.5 port vector spectrometer is a Polyvinyl chloride (PVC) sample. The thickness measured with a caliper is $1.01 \pm 0.01 \mathrm{~mm}$. The data analysis containing all the calculations and minimization routines, is performed by executing an in house developed Matlab code.

The geometric thickness of the sample determined by the $\mathrm{THz}$ measurement with the 1.5 port vector spectrometer is $1.0166 \mathrm{~mm}$, in excellent agreement with the thickness determined by the caliper measurement. The results obtained for this sample in terms of refractive index and absorption coefficient is depicted in Figure 3 and Figure 4 respectively. The refractive index is found to be weakly decreasing with the frequency with a value of 1.612 at $1 \mathrm{THz}, 1.577$ at $2 \mathrm{THz}$ and 1.553 at $3 \mathrm{THz}$. Whereas the absorption coefficient increases with frequency with a value of $18 \mathrm{~cm}^{-1}, 48.56 \mathrm{~cm}^{-1}$ and 58.15 $\mathrm{cm}^{-1}$ at 1,2 and $3 \mathrm{THz}$, respectively. Figure 3 also shows refractive index calculated for the same sample using only reflection $\mathrm{THz}$ TDS without phase correction for relative position of sample and mirror. It is less accurate and has less bandwidth than the 1.5 port spectrometer.

\section{CONCLUSION}

We conclude that this 1.5 port spectrometer is feasible to measure the refractive index, absorption coefficient and physical thickness of strongly absorbing materials accurately.

We acknowledge the European research council for funding the ERC StG "Pho-T-Lyze", Grant no. 713780 and the Deutsche Forschungsgemeinschaft for funding project PR1413/3-1.

\section{REFERENCES}

[1]. L. Duvillaret, F. Garet, and J. L. Coutaz, "Highly precise determination of optical constants and sample thickness in terahertz time-domain spectroscopy.," Appl. Opt., vol. 38, no. 2, pp. 409-415, 1999.

[2]. T. D. Dorney, R. G. Baraniuk, and D. M. Mittleman, "Material parameter estimation with terahertz time-domain spectroscopy," J. Opt. Soc. Am. A, vol. 18 , no. 7 , p. 1562, Jul. 2001.

[3]. S. Nashima, O. Morikawa, K. Takata, and M. Hangyo, "Measurement of optical properties of highly doped silicon by terahertz time domain reflection spectroscopy," Appl. Phys. Lett., vol. 79, no. 24, pp. 3923-3925, 2001.

[4]. M. Bernier, F. Garet, and J. L. Coutaz, "Precise determination of the refractive index of samples showing low transmission bands by $\mathrm{THz}$ timedomain spectroscopy," IEEE Trans. Terahertz Sci. Technol., vol. 3, no. 3, pp. 295-301, 2013.

[5]. A. Pashkin, M. Kempa, H. Němec, F. Kadlec, and P. Kužel, "Phasesensitive time-domain terahertz reflection spectroscopy," Rev. Sci. Instrum., vol. 74, no. 11, pp. 4711-4717, Nov. 2003.

[6]. A. Taschin, P. Bartolini, J. Tasseva, and R. Torre, "THz time-domain spectroscopic investigations of thin films," Measurement, Jun. 2017.

[7]. U. Nandi, J. C. Norman, A. C. Gossard, H. Lu, and S. Preu, "1550-nm Driven ErAs:In(Al)GaAs Photoconductor-Based Terahertz Time Domain System with $6.5 \mathrm{THz}$ Bandwidth," J. Infrared, Millimeter, Terahertz Waves, vol. 39 , no. 4, pp. 340-348, Apr. 2018. 
(C) 2018 IEEE. Personal use of this material is permitted. Permission from IEEE must be obtained for all other uses, in any current or future media, including reprinting/republishing this material for advertising or promotional purposes, creating new collective works, for resale or redistribution to servers or lists, or reuse of any copyrighted component of this work in other works.

Published article: Fahd R. Faridi, Uttam Nandi, Sascha Preu, 1.5 Port Vector Spectrometer for Terahertz Time Domain Spectroscopy. In: 2018 43rd International Conference on Infrared, Millimeter, and Terahertz Waves (IRMMW-THz). IEEE, 2018. S. 1-2.

DOI: $\underline{10.1109 / \text { IRMMW-THz.2018.8510415 }}$ 